\title{
There may be a way to say "NO" to gastric injury associated with the combined use of aspirin and cyclooxygenase-2 inhibitors
}

\author{
Jennifer J Williams MD, Paul L Beck PhD MD FRCPC
}

Wallace JL, Zamuner SR, McKnight W, et al. Aspirin, but not NO-releasing aspirin (NCX-4016), interacts with selective COX-2 inhibitors to aggravate gastric damage and inflammation. Am J Physiol Gastrointest Liver Physiol 2004;286:G76-81.

\section{BACKGROUND}

Due to evidence that it reduces the frequency of cardiac events and stroke, the use of daily low-dose aspirin has dramatically increased over the past 20 years (1). The use of other nonsteroidal anti-inflammatory drugs (NSAIDs) for the relief of pain and inflammation has also increased (2). Unfortunately, aspirin and conventional NSAIDs such as diclofenac are notorious for causing significant damage to the upper gastrointestinal tract because they suppress prostaglandin $(\mathrm{PG})$ synthesis (3).

Selective cyclooxygenase (COX)-2 inhibitors, such as celecoxib, were initially praised for their ability to exert analgesic and anti-inflammatory properties, while causing minimal gastric mucosal injury (3). More recent evidence, however, has demonstrated significant gastrointestinal risk. In fact, selective COX-2 inhibitors interfere with ulcer healing to the same extent as traditional NSAIDs (3).

Furthermore, as the population ages, the number of persons taking both aspirin and a selective COX-2 inhibitor is increasing (3). Both animal and human studies have demonstrated that coadministration of these medications results in significantly more severe gastric injury than either agent alone (4).

The search has begun for medications that possess cardioprotective, analgesic and anti-inflammatory properties without damaging the gastric mucosa. Aspirin compounds to which a nitric oxide $(\mathrm{NO})$ moiety has been attached seem to provide equivalent or superior cardioprotection with diminished gastric toxicity.

\section{ARTICLE SUMMARY}

Aspirin exerts protective effects on the stomach via the production of 15(R)-epi-lipoxin $\mathrm{A}_{4}$ (also known as aspirin-triggered lipoxin or ATL), which is triggered by the acetylation of COX. Selective COX-2 inhibitors, however, block the production of ATL and, therefore, exacerbate aspirin-induced gastric damage.

While the coadministration of aspirin and a selective COX-2 inhibitor is known to cause more gastrointestinal damage than either agent alone, the effects of coadministration of NCX-4016 (a NO-releasing aspirin) and a selective COX-2 inhibitor had not previously been studied. The goals of the study were twofold: to examine the effects of coadministration of NCX-4016 and a selective COX-2 inhibitor on gastric injury and inflammation, and to determine the effects of NCX-4016 on COX-2 expression (assessed by Western blot analysis and evaluation of PG and ATL synthesis) and gastric neutrophil infiltration (assessed by measurement of myeloperoxidase $[\mathrm{MPO}]$ activity).

To achieve these goals, rats were given aspirin $(10 \mathrm{mg} / \mathrm{kg}$, $30 \mathrm{mg} / \mathrm{kg}$ or $100 \mathrm{mg} / \mathrm{kg}$ ), equimolar doses of NCX-4016 or vehicle. Gastric damage, both macroscopic and histological, was scored blindly. MPO activity, PG and COX-2 expression were also determined. In the second part of the study, rats were treated with aspirin, NCX-4016 orally and either vehicle or celecoxib intraperitoneally.

As expected, aspirin induced gastric damage in a dosedependent manner. On the other hand, equimolar doses of NCX-4016 did not produce detectable gastric injury. The two agents produced comparable degrees of inhibition of PG synthesis. Celecoxib administration alone did not produce gastric damage, but coadministration of aspirin and celecoxib resulted in significantly more gastric injury than aspirin alone. Coadministration of NCX-4016 and celecoxib, however, did not produce detectable damage.

Celecoxib did not affect PG levels. An increase in MPO was seen with coadministration of celecoxib and aspirin, but not with the COX-2 alone or with NCX-4016. Aspirin, but not 
NCX-4016, administration resulted in an increased expression of COX-2. Finally, aspirin, given alone or in combination with the COX-2 inhibitor, increased the synthesis of ATL. Neither celecoxib nor NCX-4016, given in isolation, was associated with increased ATL production.

\section{COMMENTARY}

The current study confirms previous findings that selective COX-2 inhibitors increase the severity of aspirin-induced gastric injury. Furthermore, NCX-4016 does not cause gastric damage when administered with or without celecoxib. The lack of damage with this medication, in contrast to aspirin, cannot be explained by ATL production in the stomach. Unlike selective COX-2 inhibitors and aspirin, NCX-4016 does not induce granulocyte infiltration. This may be a result of its inhibitory effect on the adherence of leukocytes to the endothelium, as reported by previous investigators (5).

The gastroprotective effects of NCX-4016, given alone or in combination with a selective COX-2 inhibitor, have been demonstrated by this and other studies (6). This fact, combined with evidence that this agent suppresses platelet function and thrombus formation as effectively as aspirin $(7,8)$, has paved the way for human studies of this medication.

In fact, two small clinical trials have confirmed these findings $(4,7)$. The first clinical trial (7) was a parallel-group, double-blind, placebo-controlled trial of 40 patients randomly assigned to seven days of treatment with NCX-4016 (400 mg or $800 \mathrm{mg}$ twice daily), an equimolar dose of aspirin (200 mg or $420 \mathrm{mg}$ twice daily) or placebo. Upper endoscopy was performed before and after treatment. They examined the basal and post-treatment platelet aggregation response to arachidonic acid and thromboxane, as well as stimulated platelet production. There was significantly less gastric and duodenal injury in patients who received NCX-4016 $(\mathrm{P}<0.0001)$. There was no significant difference between the two groups in the inhibition of platelet aggregation, serum thromboxane or platelet generation. In the second clinical trial (4), 32 volunteers were randomly assigned to receive a two-week treatment with either NCX-4016 (800 mg twice daily) or aspirin (100 mg daily), alone or in combination with celecoxib (200 mg twice daily). Mucosal injury, assessed by endoscopy, was significantly more severe with aspirin than with NCX-4016 ( $<<0.01)$. Coadministration of celecoxib increased gastric injury in patients who also took aspirin, but not in those given NCX-4016. Aspirin and NCX-4016 led to comparable suppression of thromboxane levels.

Animal studies (3) have shown that COX-inhibiting $\mathrm{NO}$ donors provoke much less gastric damage but have comparable or superior analgesic and anti-inflammatory properties compared with traditional NSAIDs.

\section{SUMMARY}

The NO-donating moiety in NCX-4016 and COX-inhibiting $\mathrm{NO}$ donors seems to provide gastric mucosal protection even when given in combination with selective COX-2 inhibitors. In addition, NCX-4016 suppresses platelet function and aggregation as effectively as aspirin. Clearly, larger clinical trials will be needed. The NO-donating agents offer promise as drugs that possess analgesic, anti-inflammatory and cardioprotective properties without gastric mucosal toxicity.

\section{REFERENCES}

1. Wallace JL, Ignarro LJ, Fiorucci S. Potential cardioprotective actions of NO-releasing aspirin. Nat Rev Drug Discov 2002;1:375-82.

2. Hamerman D. Clinical implications of osteoarthritis and ageing. Ann Rheum Dis 1995;54:82-5.

3. Perini R, Fiorucci S, Wallace JL. Mechanisms of nonsteroidal anti-inflammatory drug-induced gastrointestinal injury and repair: A window of opportunity for cyclooxygenase-inhibiting nitric oxide donors. Can J Gastroenterol 2004;18:229-36.

4. Fiorucci S, Santucci L, Wallace JL, et al. Interaction of a selective cyclooxygenase- 2 inhibitor with aspirin and NO-releasing aspirin in the human gastric mucosa. Proc Natl Acad Sci USA 2003; 100:10937-41.

5. Fiorucci S, Distrutti E, Mencarelli A, et al. Cooperation between aspirin-triggered lipoxin and nitric acid (NO) mediates antiadhesive properties of 2-(Acetyloxy) benzoic acid 3(nitrooxymethyl) phenyl ester (NCX-4016) (NO-aspirin) on neutrophil-endothelial cell adherence. J Pharmacol Exp Ther 2004;309:1174-82.

6. Fiorucci S, de Lima OM Jr, Mencarelli A, et al. Cycloxygenase-2derived lipoxin A4 increases gastric resistance to aspirin-induced damage. Gastroenterology 2002;123:1598-606.

7. Fiorucci S, Santucci L, Gresele P, Faccino RM, del Soldato P, Morelli A. Gastrointestinal safety of NO-aspirin (NCX-4016) in healthy human volunteers: A proof of concept endoscopic study. Gastroenterology 2003;124:600-7.

8. Wallace JL, Muscara MN, McKnight W, Dicay M, Del Soldato P, Cirino G. In vivo antithrombotic effects of a nitric oxide-releasing aspirin derivative, NCX-4016. Thromb Res 1999;93:43-50. 


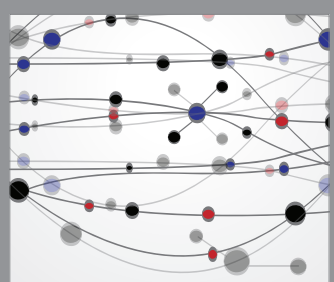

The Scientific World Journal
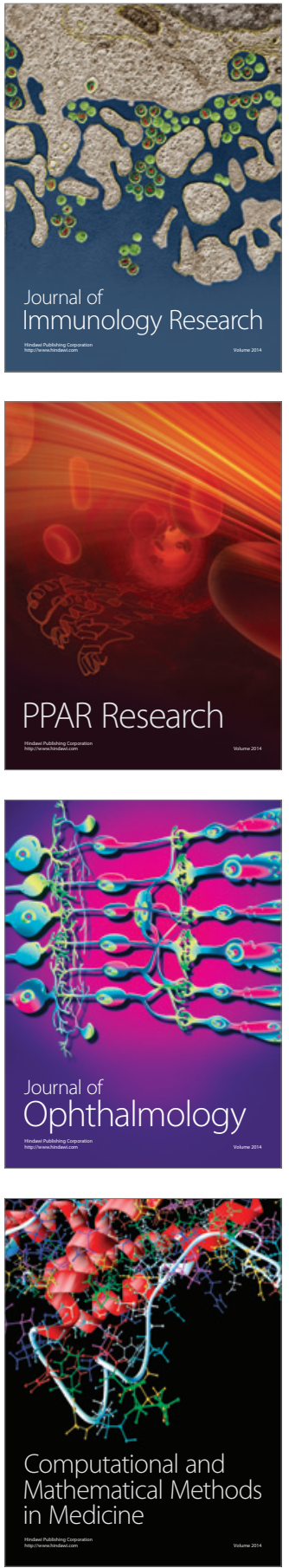

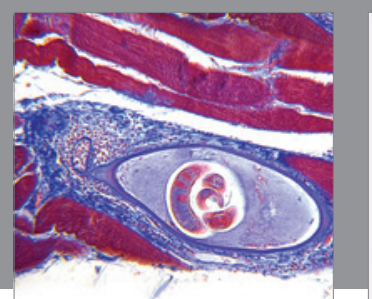

Gastroenterology Research and Practice

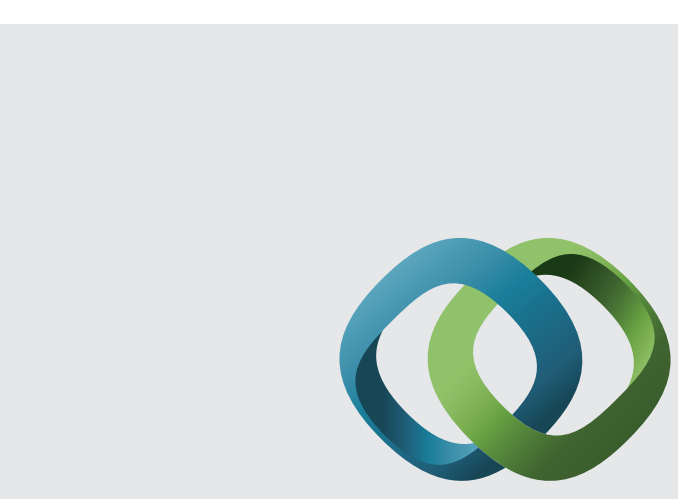

\section{Hindawi}

Submit your manuscripts at

http://www.hindawi.com
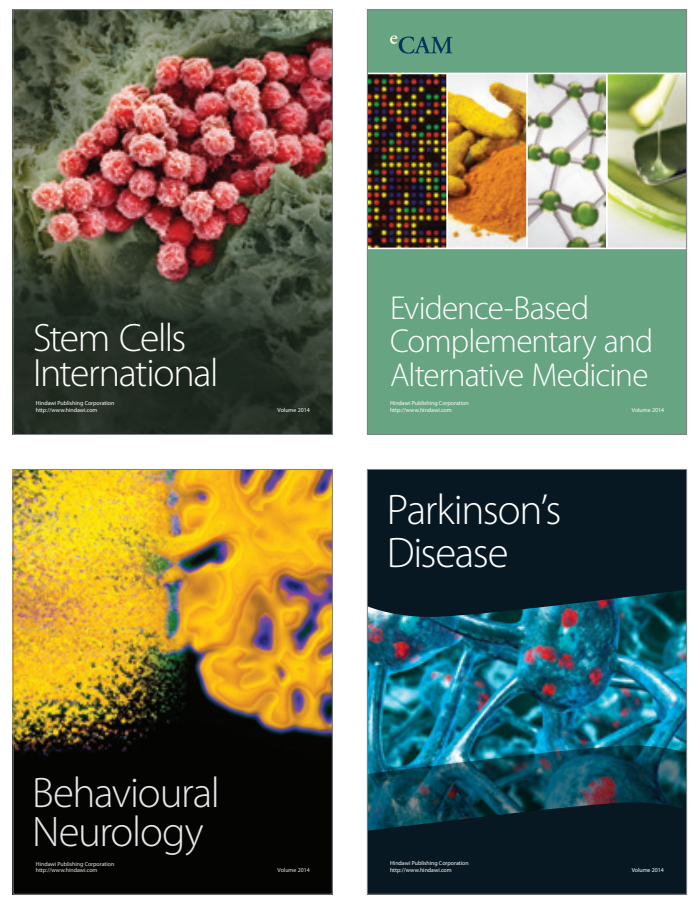
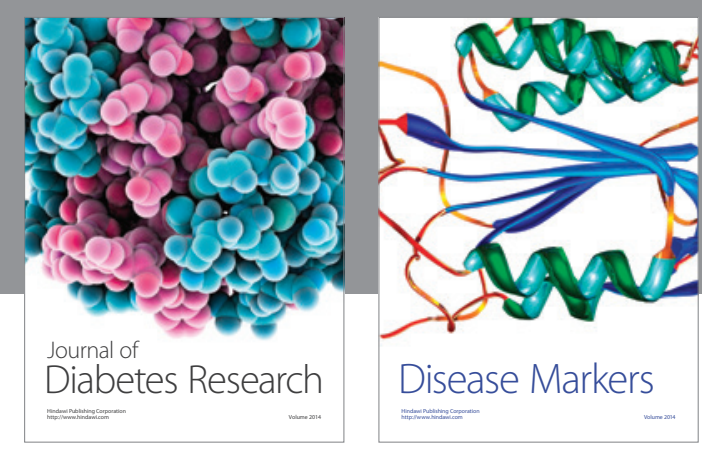

Disease Markers
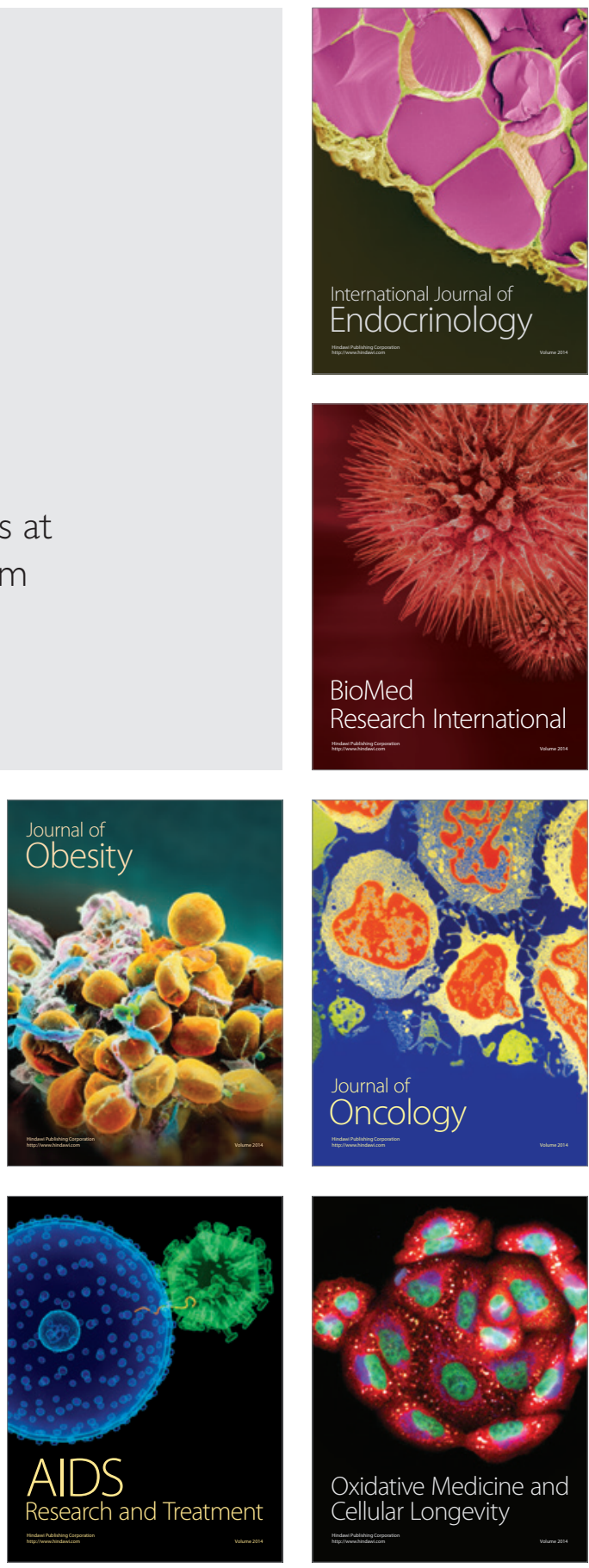